\title{
A model to integrate pathology teaching using technology
}

\author{
Chumba $D^{1}$, Macharia $B^{1}$, Smith $\mathrm{J}^{2}$, Wade $\mathrm{J}^{2}$
}

\begin{abstract}
Purpose: Use of technology to integrate pathology teaching in the third year of medical training. The teaching of pathology and disease includes several sections that have been taught by separate departments of immunology, haematology, microbiology, histopathology and biochemistry. It has been noted that students are not able to integrate the teaching from all these departments. To address this problem we have developed a model to integrate the teaching of the different sections by providing additional cases and materials in the computer laboratory.
\end{abstract}

Methods: The eight week course of general pathology has been chosen for this model as it is the first section of pathology and the availability of teaching materials in the server of the computer. Three practical sessions are given to each section, histopathology, microbiology, haematology, immunology and clinical chemistry. Additional information was made available to students in the new thin client computer laboratory. A questionnaire was administered at the end of the course to half of the students. The responses which targeted in three areas, content, relevance, and acceptance and analyzed.

Results: The eight week course was received enthusiastically by the students. The data indicated that the students generally accepted a computer-based instruction in teaching pathology. There was no improvement in performance of the class at the end of the term examinations as compared to the previous year.

Conclusions: Computer-based instruction is possible in low resource countries. Use of technology can address challenges relating to integration of teaching. This model could serve as a nucleus for more extensive restructuring of teaching in the entire Medical School as well in other Medical Schools in Kenya and the rest of Africa.

Keywords: computer-based instruction; pathology, problem based learning

\section{Introduction}

Tomorrow's doctors' was published in the Europe in 1990s (General medical council, 1993) and led to major curricular reorganization in most European medical schools. It is now being conceptualized in African medical schools. There has been much discussion about what methods we should be using, and

\footnotetext{
1. Moi University School of Medicine (Kenya)

2. Indiana University School of Medicine (USA)

Corresponding author:

Chumba D.

Moi University School of Medicine,

MTRH building Nandi Road,

P.O Box 4606 Eldoret Kenya

E-mail address:dchumba@yahoo.com, infoingolan@gmail.com
}

where they would fit in the curriculum (Lowry, 1993) The cellular and molecular processes encompassed in pathology provided the basis for the understanding of clinical medicine. Clinical pathology, including the main disciplines of microbiology, immunology, haematology, and chemistry, underpin the practice of clinical medicine. Pathology should be a popular subject with students because it has an immediate relevance to medicine. In many African medical schools it is not popular. This may be related to teaching methods and poor integration among pathology disciplines.

Pathology teaching in most African medical schools has been based on lectures and practical. Moi University School of Medicine has adopted problem based learning as the main mode of teaching with good success. With the improvement of internet connectivity and collaboration with other international 
universities, students have been able to access technology. The interaction is either by exchange program with Indiana University or web- based with Loyola University through AMEN-Kenya web site.

Teachers are often unable to motivate students to learn various aspects of pathology. This is due in part to changing patterns of student teaching method preferences and teacher apathy. Computer based instruction in itself motivates students to learn. Computer based instructions has not been available in most low income countries, especially in Africa. Moi University School of Medicine has divided pathology into five (5) sections, immunology, haematology, microbiology, histopathology and biochemistry. This division has brought problems of integration across the sections. This has made this very important subject poorly understood by students.

Pathology can be taught in a variety of ways: lectures, demonstrations, practical classes, seminars, and discussion groups as in PBL. In addition, there is an increasing number of computer based programs of instruction available. Students learn best when they are interested, can see the relevance of what they are learning, and are intrinsically motivated.

Introducing elements of discussion and interaction, especially through the use of clinical examples and problem solving practical's, will help maintain interest. The use of visual aids, computer based programs, knowledge checking, and summarizing enhances student learning and understanding. Ritual humiliation which is common in many African schools does not enhance student learning.

Pathology is a subject with a vast and ever expanding knowledge base. It is thus more important than ever that pathology learned in medical curriculum is relevant, emphasizes the understanding of mechanisms and principles rather than detailed facts. As students continue their medical education more detailed systemic pathology will become more relevant and appropriately learned. The course of general pathology explains the basic alterations in diseases.

The document Tomorrow's Doctors (General medical council, 1993) recommended that medical education should foster 'learning through curiosity, and exploration of knowledge and the critical evaluation of evidence 'Moi University was established in the 1990's as the second medical school in Kenya. At that time no lecturer teaching pathology was willing to leave the primary medical school in Nairobi and join Moi University; hence, there was a major shortage of lecturers. Therefore, expatriates were recruited and most were retired professors. The introduction of problem-based learning (PBL) was not accepted by most. Divisions among the various departments teaching pathology disciplines soon became a major hindrance to teaching. The school, in trying to address the problem created the five departments named earlier. Each department started teaching their courses from their own perspective and clinical cases were not taught. Students were learning pathology sections in an uncoordinated manner.

This problem was noticed when external examiners found that students especially the weak ones could not effectively integrate the various aspects of pathology. If a haematologist asked the question the students would answer it and relate to haematology even if the most important discussion in the case was microbiology.

To address this problem and not to produce conflict with faculty members, cases were introduced in the new thin client computers laboratory. Cases obtained from Indiana School of Medicine in USA were put in a server which was connected to sixty terminals which is appropriate fan half the class at a time.

The traditional PC functions as a desk top device at the users' desk and handles input from the keyboard and mouse, displays program output, stores the users data and runs the users programmes.

A computer laboratory, on the other hand, separates these functions: the device at the user's desk only handles the keyboard and mouse input and displays the program output. The user's program actually executes his/her which to data is stored on the central server.

Because the thin clients have no operating system (any more than, say, a printer has an "operating system"), maintenance on the desktop device is virtually eliminated (Dominis et al, (1999). Rather, system managers need to focus only on configuring and securing the one server rather than 60 desktop computers. The medical school configured our laboratory so that the thin clients do not have access to the web. While this constrains students to using only the teaching materials we have 
placed on the server, this configuration greatly reduces the risk of computer virus infection. Further, the thin clients are configured so students may not use them for games, web surfing, or personal communications. While this means that students do not have access to a number of good pathology web sites, we feel that having a student self-study computer system is highly available and low maintenance outweighs this inconvenience.

These characteristics of thin clients are inexpensive to install and operate and suitable for low resource institutions. Computer teaching would otherwise be too expensive to install and operate in most third world countries.

\section{Statement of the problem}

This abstract follows three consecutive negative comments by external examiners who noted that there was disjointed knowledge of pathology by students, even, the very bright ones. After extensive search for what could be the problem, pathologists sought information from those who are quite established in PBL. Issues raised were discussed and the main problem was noted for integration. Integration in pathology courses would optimally include basic sciences; however, in this paper integration was across sections of pathology only. Basic sciences shall be considered in the future.

Moi School of Medicine in its curriculum starts teaching pathology in the third year of a six year medical education curriculum. Pathology is covered in three terms equivalent to 46 units. Pathology starts with general pathology which teaches basic pathological processes and it's their effects on biochemical, morphological, haematological and immunological factors. Introduction to toxicity is also covered. Most of these pathological processes will be repeated again during the systemic pathology. Due to its importance eight weeks has been given to this course while the others is given 4 or 5 weeks.

Three months before the course of general pathology started the school had installed the thin client computer system. Limited teaching resources had been installed in the server; however, there were a few relevant cases addressing all the areas of pathology, i.e. haematology, histopathology, microbiology, clinical chemistry and immunology. The topics addressed include, cell injury, inflammation, genetic disorders and Neoplasm.

\section{Methods}

The course coordinator for general pathology had attended the course director's workshop in IAMSE (International Association of Medical Science Educators). In the pathology course at MUSM, he allocated time which was meant for self-directed learning (SDL) to be spent in the computer laboratory. Each group of forty to sixty students were given two to three hour sessions in which they had free access to materials in the server. Two tutors were in the computer laboratory during that period to guide and answer any questions. An attendance list was signed by those who came. This was not mandatory, but every other student was given a questionnaire to complete. A total of 40 questionnaires were given out and 39 were returned

\section{Teaching Facilities}

The thin client computer system was bought and installed with the funds obtained from Prof. James Smith (Emeritus Professor, Indiana University). The server was installed in a safe room at the Department of Anatomy, School of Medicine Moi University. The server is connected to 60 monitors with a key board. No access to the internet is allowed. The computer lab is shared with the Department of Anatomy. The computer lab is open to students twice a week (Thursday $10 \mathrm{am}-1 \mathrm{pm}$ and Monday $2-5 \mathrm{pm}$ ) a total of 6 hours per week and also during any scheduled seminars. The main questions addressed were:

1. Could the use of computer based teaching methods be possible in poor resource countries?

2. Are these methods acceptable to students and can they change the study habits of medical students at MUSM?

3. Will access to this computer based teaching improve performance on standardized exams compares to the previous class which did not have access to this computer lab?

4. How readily will students accept computer based teaching methods?

Standard end of term examinations were set as had been done in previous year. A pool of questions most of which were derived from GRIPE (Group for Research in Pathology Education) resources were selected within the Department by the lecturers in the department of Human Pathology and Forensic Medicine. The other departments teaching pathology 
sections i.e. immunology, biochemistry, microbiology and haematology were not aware of what we were doing, except that they had wondered why the SDL time had been used for computer lab.

A questionnaire was given to every other student. Three areas were addressed in the questionnaire (1) Acceptance (2) Adequacy
(3) Content. Results of end of term exams (ETE1) was obtained from all the sections of pathology except haematology where the exam will be done in second term (ETE2). The best score, average score and the lowest score in percentage was calculated and analyzed.

Figure 1: Use of computer lab

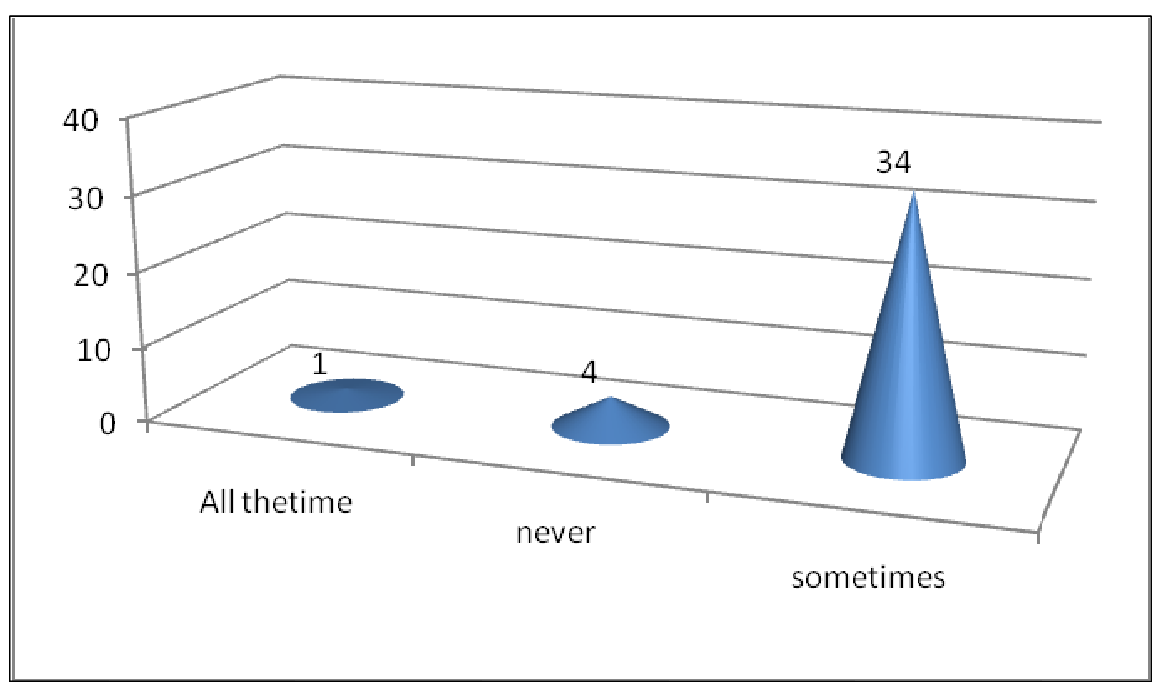

Question: How often did you use the computer lab during the course of general pathology? Figure 1 shows that about $90 \%$ of the students used the materials that were provided and only $10 \%$ never used.

Figure 2: Teaching materials

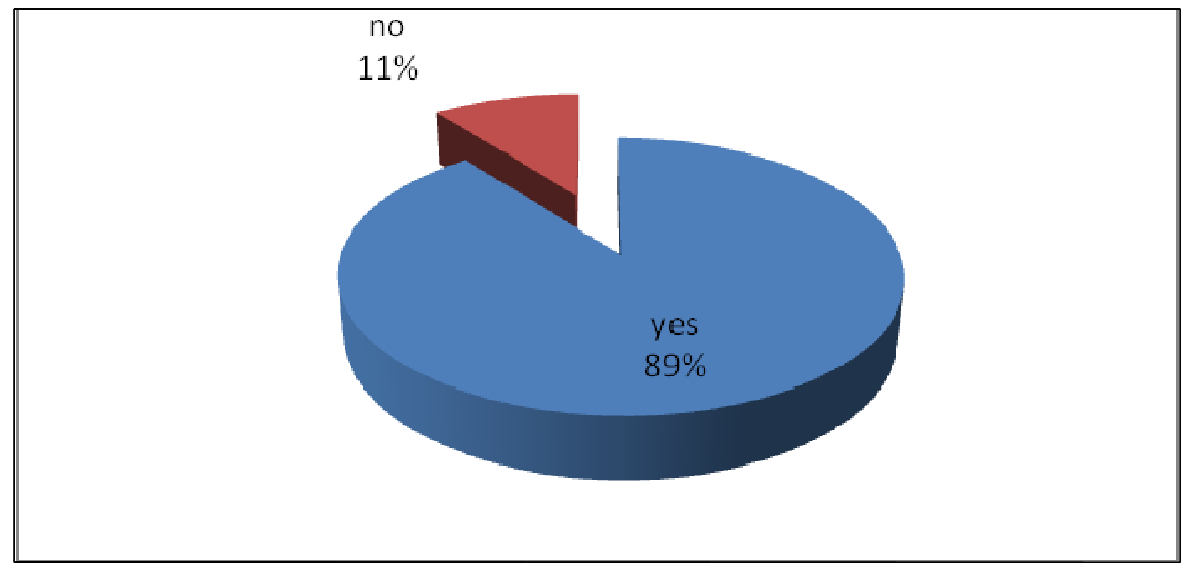

Question: Did you feel the teaching materials in the computer lab helped you in any way to learn and integrate the various sections of pathology?

Figure 2 shows that $89 \%$ of the students thought that the materials helped them integrate pathology teaching and $11 \%$ said no 
Figure 3: Contents of the server

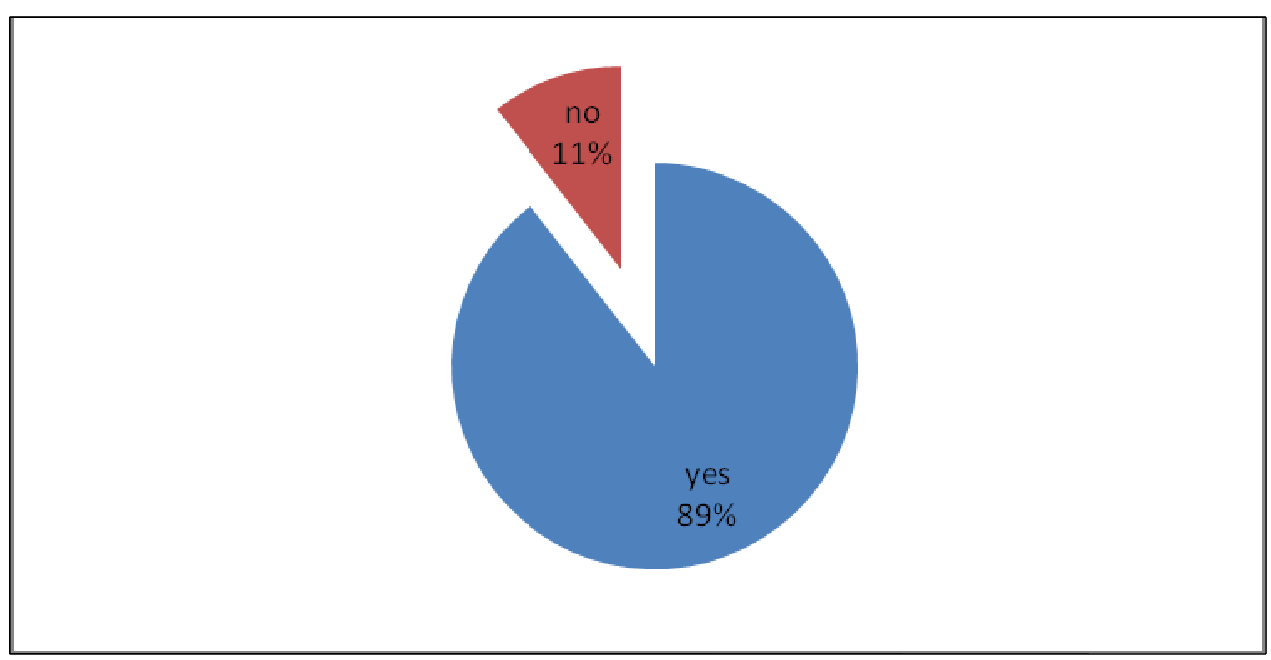

Question: Do you think the contents server helped you to learn the course of general pathology (MSP 300). Figure 3 shows that $89 \%$ of the students thought that the contents of the materials provided helped them learn pathology

Figure 4: Adequacy of the contents in the serve

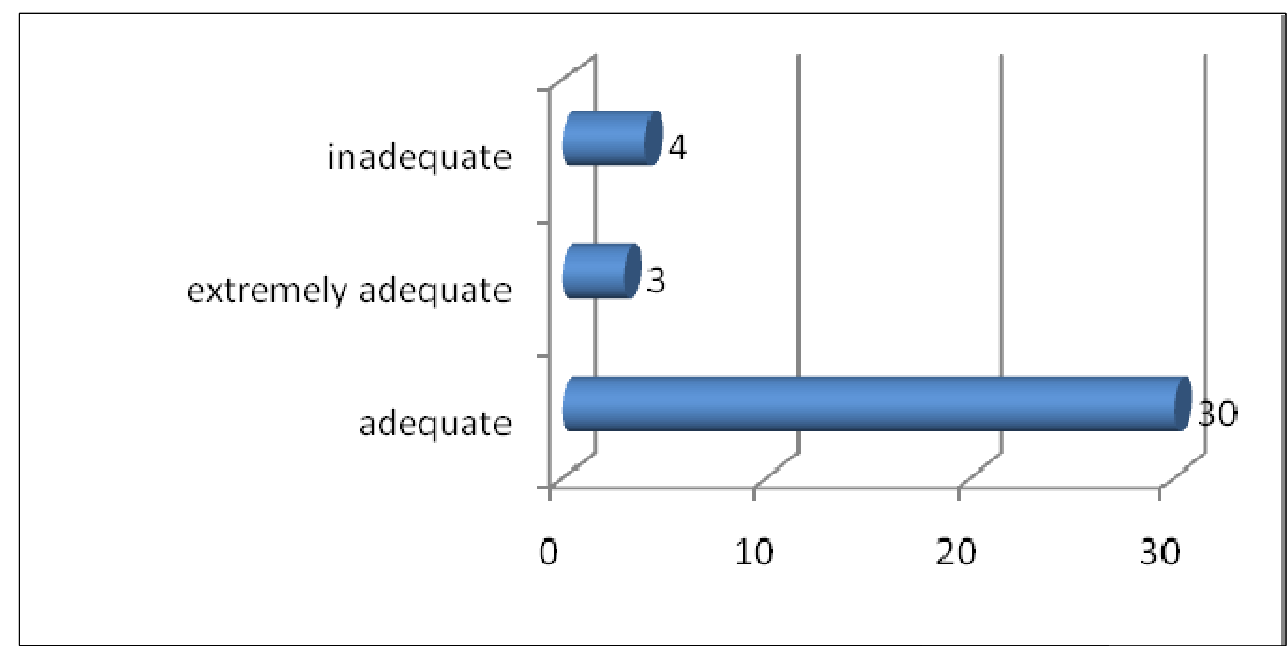

Figure 4 shows that $77 \%, 11 \%$ think that it was inadequate and 5\% thought it was extremely useful. 
Figures 5: Rank the overall importance of the computer lab in teaching pathology.

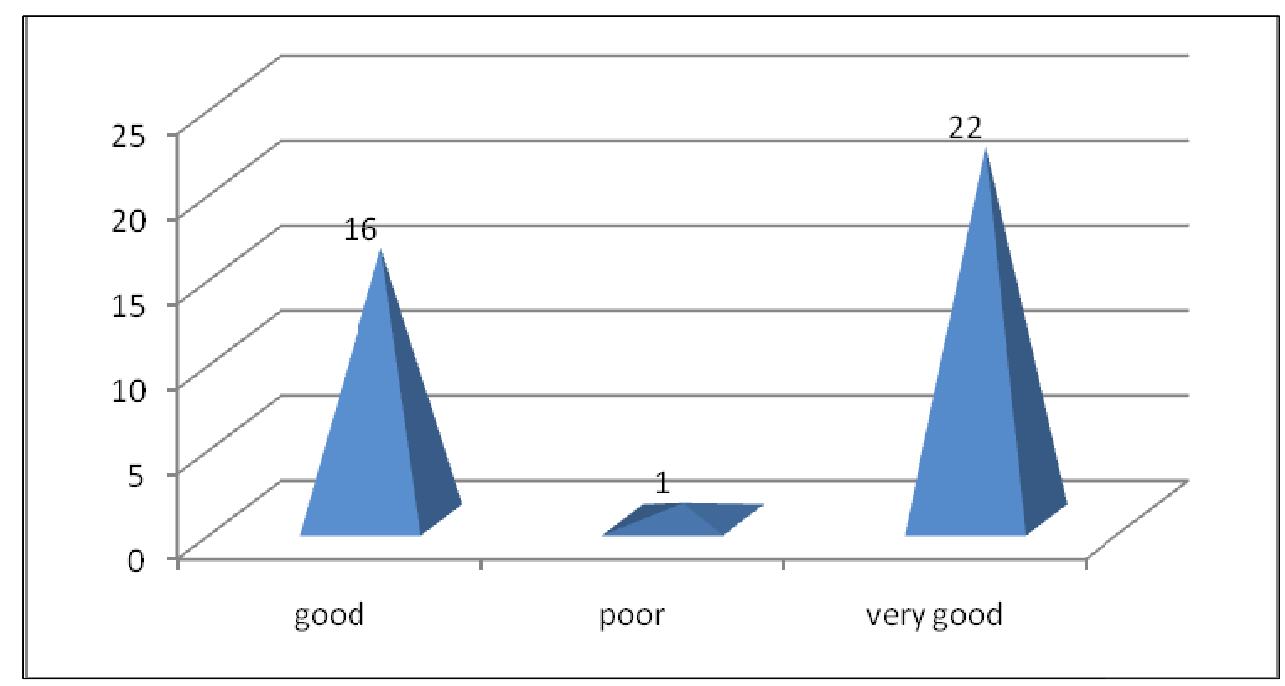

Figure five shows that most students think that technology is important in teaching pathology

\section{Discussion}

The data was collected on use of the computer lab during the general pathology course. The full effects of this will be seen at the end of year when external examiners give their report; however, these results reflect obvious enthusiasm for the use of computer based teaching methods. The investment to establish the program was not substantial and can be afforded by most third world country universities. Private funding and interest was an important part of the process which otherwise would not have been initiated by the school.

A model like this could serve as a nucleus for more extensive changes and the restructuring of teaching in the entire MUSM as well as in other universities in Kenya and the rest of Africa. At the present time, it is not possible to determine whether the computer based learning system has had a quantifiable impact on the scholastic performance of students as seen in the performance of ETE 1. The fact that there was a general poor performance by this class as compared to last year may just mean that this class is a weaker class than last year. The type of exam were not to test integration as It was mainly oral as in the clinical years rather than recall type of questions given in ETE 1.

While, the preliminary data were encouraging, long term studies must be done to see the overall impact of this type of instruction and to know the impressions of the instructors teaching the courses as a whole.
This report is a testimony that the enthusiasm for the computer based learning is as fervent in Kenya as in other countries. The students seem to be enthusiastic about this new technology even though computers are not expected to solve all the problems as seen in the performance of ETE 1 exams. This development is certainly an impetus to improve medical education and develop good working habits, and a foundation for lifelong continuous education among students.

\section{References:}

General medical council (1993) Tomorrow's doctors recommendations on undergraduate medical education. London: GMC.

Lowry S (1993) Medical Education. London: BMJ Publishing Group, pp.498-503.

Bligh, J. (1995) Problem based, small group teaching, Br Mrd J, 311, pp. 342-343.

Mara, Dominis., Marin, Nola. \& Stanko, Jukiæ. et al. (1999) Computer-Based Teaching of Pathology at the Zagreb University School of Medicine. Croatian Medical Journal, 40, 3, March.

Mean Time between Failure (MTBF) of the Oracle Sun Ray thin client is greater than twenty years. Source: Oracle Sun Ray 3 Client Data Sheet; Available at: http://www.oracle.com/ us/technologies/virtualization/sunray-3-clientds-173275.pdf. 